\title{
Sensing atmospheric structure: Tropospheric tomographic results of the small-scale GPS campaign at the Onsala Space Observatory
}

\author{
A. Flores $^{1 *}$, L. P. Gradinarsky ${ }^{2}$, P. Elósegui ${ }^{3}$, G. Elgered ${ }^{2}$, J. L. Davis ${ }^{3}$, and A. Rius ${ }^{1}$ \\ ${ }^{1}$ Institut d'Estudis Espacials de Catalunya, IEEC-CSIC, Spain \\ ${ }^{2}$ Onsala Space Observatory, Chalmers University of Technology, Sweden \\ ${ }^{3}$ Harvard-Smithsonian Center for Astrophysics, MA, U.S.A.
}

(Received December 31, 1999; Revised June 19, 2000; Accepted August 2, 2000)

\begin{abstract}
Tropospheric tomography using data from local networks of Global Positioning System (GPS) receivers is producing encouraging spatio-temporal representations of the wet refractivity field. In this work we present the results from a small-scale geodetic experiment that we carried out at the Onsala Space Observatory. Seven GPS receivers distributed within a radius of $3 \mathrm{~km}$ from the center, were deployed during 21 days in the summer 1998. The limited number of sites and their spatial configuration present a challenge for tropospheric tomography. Using novel GPS techniques to determine the vertical structure of the atmosphere, we observed, for one session, a strong horizontal water-vapor gradient with a leading edge at higher altitude than the trailing edge, entering from the north. The vertical structure obtained independently using tomographic techniques matched such situation. These results suggest tomography is a promising technique for the determination of the spatio-temporal structure of the atmosphere. We will present preliminary results of the tropospheric tomography, using simulations and experimental data, together with some comparisons with radiosonde data.
\end{abstract}

\section{Tropospheric Tomography Equations}

The effect of the atmosphere on GPS signals is an extra delay which depends on the refractivity along the slant path of each ray as: $L_{t}=\int_{\text {s.l. }} 10^{-6} \mathrm{Ndl}+\mathcal{S}-\mathcal{G}$, where refractivity may be written as $N=77.6 \frac{P}{T}+3.776 \cdot 10^{5} \frac{P_{w}}{T^{2}}+1.4 \mathrm{~W}=$ $N_{h}+N_{w}+1.4 W$, where $P$ is the pressure in mbar, $P_{w}$ is the water vapor pressure in mbar, $T$ is the temperature in $\mathrm{K}$, and $W$ is the liquid water vapor in the atmosphere in grams per cubic meter (see Thayer, 1974; Kursinski, 1997). The latter term is generally neglected. The term $\mathcal{S}-\mathcal{G}$ is the bending term which is about $1 \mathrm{~cm}$ or less for elevations greater than $15^{\circ}$ and in general will not be considered (see Bevis et al., 1992). This atmospheric delay may be modeled as a zenith component plus horizontal gradients. Each of them can be further split into hydrostatic (due to the neutral gases of the atmosphere) and wet (due to the dipolar part of water vapor) components:

$$
\begin{aligned}
L_{t}= & m_{h}(e)\left[L_{z}^{h}+\cot e\left(\vec{L}_{h}^{G} \cdot \hat{\rho}(\phi)\right)\right] \\
& +m_{w}(e)\left[L_{z}^{w}+\cot e\left(\vec{L}_{w}^{G} \cdot \hat{\rho}(\phi)\right)\right]
\end{aligned}
$$

where $L_{z}^{h}$ and $L_{z}^{w}$ are the hydrostatic and wet zenith delays, $e$ and $\phi$ are the satellite elevation and azimuth as seen from the station, respectively; $\hat{\rho}(\phi)$ is the horizontal unit vector

*Present address: IEEC, Edif. Nexus, 204, Gran Catpita 2-4, 08034 Barcelona, Spain.

Copy right $(\mathrm{C})$ The Society of Geomagnetism and Earth, Planetary and Space Sciences (SGEPSS); The Seismological Society of Japan; The Volcanological Society of Japan; The Geodetic Society of Japan; The Japanese Society for Planetary Sciences. defining the direction of the projection of the ray on the horizontal plane; $\vec{L}_{h}^{G}$ and $\vec{L}_{w}^{G}$, are the hydrostatic and wet delay gradients; $m_{h}$ and $m_{w}$ are the hydrostatic and wet mapping functions.

When applying tomographic techniques to retrieve the spatio-temporal structure of the water vapor in the lower troposphere, one needs observations of the Slant Wet Delays (SWD). To this end, it is necessary to remove from the solution the hydrostatic zenith delay (ZHD) and the horizontal hydrostatic gradients (HHG). The ZHD can be removed using surface pressure measurements, as described in Elgered et al. (1991). The delay gradient parameter $\vec{L}^{G}(t)$ in Eq. (1) has units of excess path length and is defined as (Davis et al., 1993):

$$
\vec{L}^{G}(t)=10^{-6} \int_{0}^{\infty} z \vec{\psi}(z, t) d z
$$

where $\vec{\psi}(z, t)=\left.\nabla_{\vec{\rho}} N(\vec{\rho}, z, t)\right|_{\vec{\rho}=0}$ is the horizontal gradient of the refractivity, $\vec{\rho}$ is the horizontal displacement vector, and $z$ is the height above the surface. As shown in Elósegui et al. (1999) and Ruffini et al. (1999) the gradient of the field of the zenith components computed at each station is

$$
\vec{G}(t)=10^{-6} \int_{0}^{\infty} \vec{\psi}(z, t) d z,
$$

has units of excess path length per unit distance, and it is a network dependent parameter. In the hydrostatic case, it can be assumed that the refractivity field follows an exponential law in the $z$ magnitude with scale height $H, \vec{N}=$ $N_{o}(\vec{\rho}) e^{-z / H}$, and in such a case (as shown in Ruffini et al. (1999), the relationship between $\vec{L}_{h}^{G}(t)$ and $\vec{G}_{h}(t)$ results in $\vec{L}_{h}^{G}(t)=H \vec{G}_{h}(t)$. Thus, the hydrostatic gradients can 
be calculated by estimating the ZHD at each site, computing $\vec{G}_{h}$, and then $\vec{L}_{h}^{G}$ (another approach is described in Bar-Sever et al. (1998) where they are considered to be constant over periods of 12 hours and hence removed by averaging the gradient solution over that period and demeaning it). Once the hydrostatic components have been modelled, they can be removed from the tropospheric estimates and the SWD are formed by mapping the zenith and gradient time-series solutions to the directions of the rays and adding the post-fit residuals to each measurement. This recreates the individual slant delay measurement.

The SWD are written in terms of the discretization of the space in voxels to form a linear system, as explained in Flores et al. (2000) and Ruffini et al. (1998). The tomographic linear system is therefore written as

$$
\left[\begin{array}{l}
\mathbf{y} \\
\mathbf{l}
\end{array}\right]=\left[\begin{array}{l}
\mathbf{A} \\
\mathbf{B}
\end{array}\right] \mathbf{x} \Longrightarrow \hat{\mathbf{y}}=\mathbf{S x} .
$$

where $\mathbf{y}$ is the vector of $\mathrm{M}$ observations (SWD), $\mathbf{A}$ is an $\mathrm{M} \times \mathrm{N}$ matrix representation the modeling of the observations and $\mathbf{x}$ is the vector of $\mathrm{N}$ unknowns. In our implementation, each ray is modeled as a finite sum of the contributions of finite-size voxels where refractivity is considered to remain constant. Thus, the element $\mathbf{a}_{\mathbf{i j}}$ contains the length of ray $i$ accross voxel $j$ and $\mathbf{x}$ represents the wet refractivity values for each voxel. $\mathbf{B}$ and $\mathbf{l}$ represent the set of equations of constraint, which define the value of a voxel as a weighted mean of its neighbours. A Kalman filtering together with smoothing process has also been implemented following Herring et al. (1990). The inversion of the linear system at each batch is done using the SVD technique as described in Press et al. (1992). The cut-off value established for the eigenvalues has been taken as in Flores et al. (2000) according to the considerations of noise and resolution there explained.

\section{The Network and the GPS Data Processing}

During three weeks in August, 1998, six additional GPS receivers were deployed close to the IGS site in Onsala. We named the campaign REGINA. Baselengths between stations ranged from $600 \mathrm{~m}$ to $4.2 \mathrm{~km}$. The purpose of REGINA was to obtain a continuous set of data for local atmospheric studies. The locations of the receivers are shown in Fig. 1.

We processed the August 24, 1998 using the GIPSYOASIS II (Webb and Zumberge, 1997) software and the LOTTOS/GIST (Flores, 1999) software package. The ZHD was extracted from pressure measurements at the IGS site. Because we only had surface pressure measurements at one location, we could not compute the $\vec{G}_{h}(t)$ and hence $\vec{L}_{h}^{G}(t)$ as described above; however, judging from the analysis using the numerical weather model NCAR Mesoscale Model $\left(\mathrm{MM}^{1}\right)$, run with a maximum resolution of $5 \mathrm{~km}$, the Onsala peninsula was free from surface pressure gradients, and we thus considered the hydrostatic gradients to be negligible.

\section{Simulation}

In order to test the possibilities of this network configuration, we first performed a simulation, keeping the location of the stations and using, as a test case, a 3D refractivity field

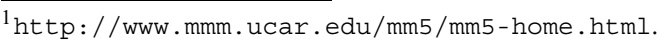

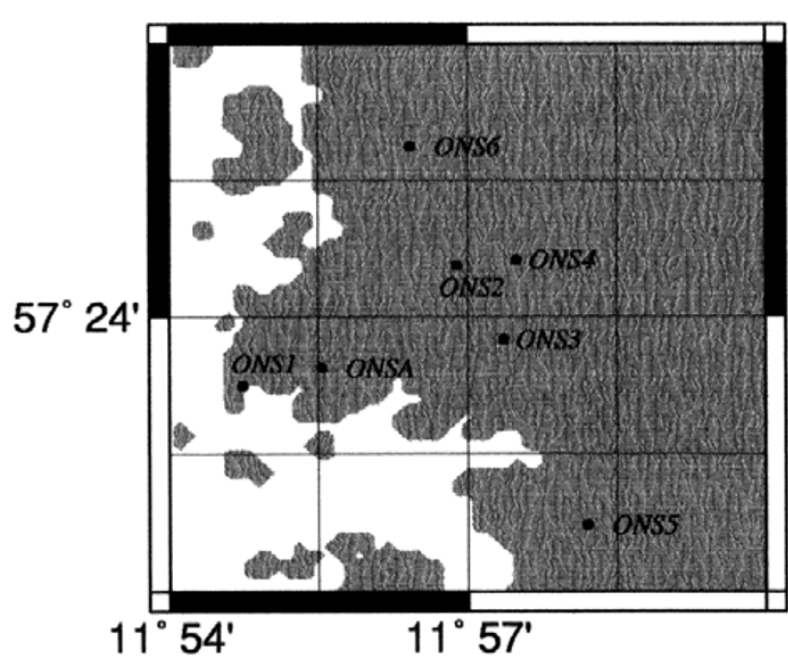

Fig. 1. Map with the location of the stations of the REGINA campaign at the Onsala Space Observatory during the summer of 1998, and representation of part of the grid used. Each voxel is $1.2 \mathrm{~km} \times 1.2 \mathrm{~km}$.

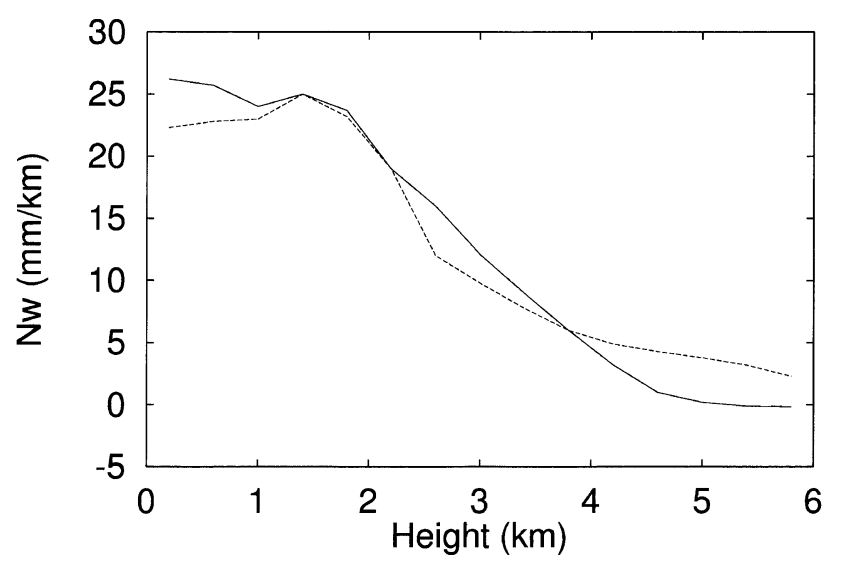

Fig. 2. Simulation results for the REGINA campaign: 3D field from radiosonde (dash) and 3D tomographic reconstruction (solid).

constructed from a radiosonde launch at Madrid on December 11998 and applying a linear gradient with north-east direction. Gaussian white noise with $\sigma=10 \mathrm{~mm}$ was added to each observation. The results are presented in Fig. 2. It can be seen that the agreement is good between both fields. The radiosonde profile shows a rather well mixed region below $1000 \mathrm{~m}$ with a relative maximum at $1400 \mathrm{~m}$. In other words, the $N_{w}$ profile traced by the radiosonde is rather constant below $1000 \mathrm{~m}$, and then peaks at $1400 \mathrm{~m}$, decaying for higher altitudes. The tomographic solution, on the other hand, shows a smoother peak at $900 \mathrm{~m}$ and the well mixed region below that. For altitudes higher than $3 \mathrm{~km}$, the tomographic solution looses resolution and smoothly decays to zero. This simulation shows that the network is sufficient to discriminate some atmospheric phenomena.

\section{Tomographic Solution for August 24, 1998}

The tomographic solution considered a $6 \times 6$ grid at each layer height (Fig. 1 shows the $4 \times 4$ grid covering the network, where each voxel is $1.2 \mathrm{~km} \times 1.2 \mathrm{~km}$; the other voxels 

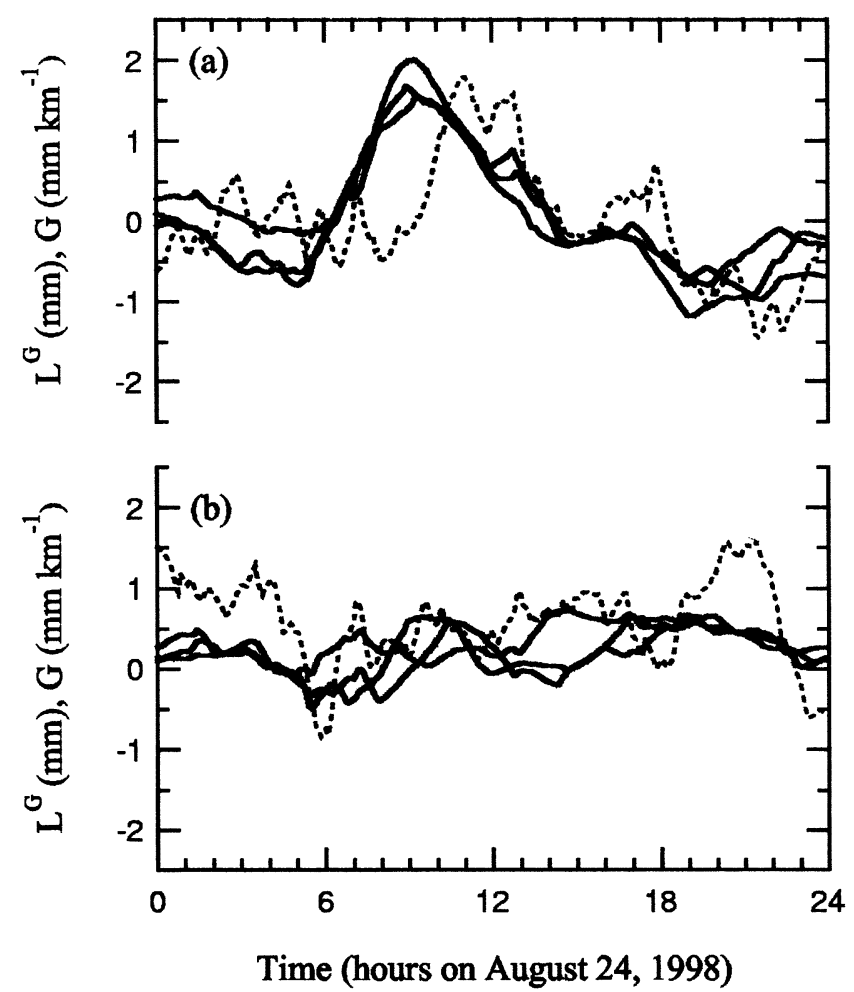

Fig. 3. Delay gradients for three stations during August 24, 1998 for the north (top) and east (bottom) components (solid). The other four stations show a very similar behaviour but have been omitted for clarity. There is a clear gradient to the north, which is two hours ahead from the corresponding $\vec{G}$ value (dashed) (from Elósegui et al., 1999).

are defined to capture the total length of the ray path below $10 \mathrm{~km}$ at the minimum elevation angle of $7^{\circ}$ ), 26 layers of $400 \mathrm{~m}$ thick and 96 batches of 15 min each. The Kalman filter was run forward and backwards with a uniform $\delta=2$ $(\mathrm{mm} / \mathrm{km}) / \sqrt{h}$; this time filtering allows us to benefit of the data from a bigger time window when no data is available for certain voxels.

A comparative analysis of the results in Elósegui et al. (1999) and the tomographic solutions has been carried out in order to verify the agreement of the solution and the interpretation given there with the tomographic results. In Fig. 3 we reproduce the time-series of $\vec{L}^{G}$ and $\vec{G}$ (dashed) for the overall network. There is a lag of 2 hours between both types, while having a high degree of correlation. In Fig. 4 we show isosurfaces of the wet refracitivity field, when it starts to enter in the analyzed region (6:00 UTC) and when it reaches the network (12:00 UTC).

There is an initial increment of the north component of $\vec{L}^{G}$ at around 6:00 UTC, but nothing is detected in $\vec{G}$. Considering Eqs. (2) and (3), $L^{G}(t)$ weights the gradients with the height at which they are located; therefore, $L^{G}(t)$ will sense a refractivity gradient entering at high altitudes. If we now look at the tomographic solution (see Fig. 4, top), we observe that a refractivity gradient is entering from the north-west, with a leading edge at around $800 \mathrm{~m}-1000 \mathrm{~m}$, at latitudes close to $57.7^{\circ}$ (note also that in Fig. 3 the east component of $\vec{L}^{G}$ is slightly negative). At 09:15 UTC (not shown) the north gradient is strong at $1200 \mathrm{~m}$ and starts to appear at
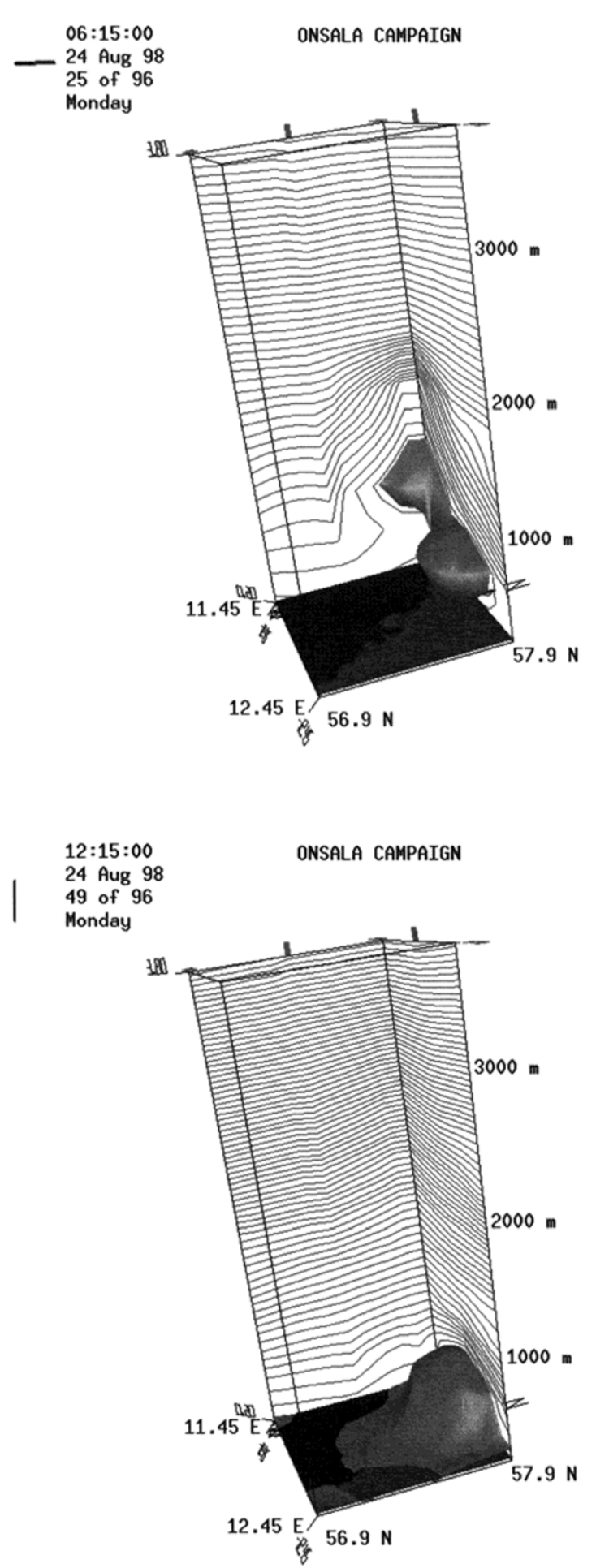

Fig. 4. Top: Tomographic solution for 06:00-06:15 UTC. Isosurface is at $17 \mathrm{~mm} / \mathrm{km}$. Bottom: Tomographic solution for 12:00-12:15 UTC. Isosurface is at $27 \mathrm{~mm} / \mathrm{km}$.

surface level; there is a strong east component of the gradient at higher altitudes within the north-east corner. Note that in Fig. 3 the north component of $\vec{G}$ starts to increase, while $\vec{L}_{N}^{G}$ peaks, and there is a noticeable positive value of $\vec{L}_{E}^{G}$. At 12:15 UTC, the gradient at low altitudes is mostly towards the north around the IGS site and again strong at 
$57.7^{\circ}$ north at $1200 \mathrm{~m}$ (see Fig. 4). Finally, the high wetter layer vanishes in the afternoon and at around 18:00 UTC it is no longer present. At that time, a small gradient is crossing from the south, as seen in the tomographic reconstruction.

It is worth mentioning that, while the tomographic reconstruction gives a richer description of the troposphere, in terms of space and time (particularly in terms of vertical distribution), the results are obviously driven by the zenith plus gradients solution of the GPS data processing: we have seen that even small gradients are reproduced by the 4D solution. Therefore, if the tomographic representation is aimed to the wet refractivity, one must correctly remove the hydrostatic gradients during the GPS data processing. For the REGINA campaign we were limited to numerical weather models to compute the hydrostatic gradients (which were seen to be negligible), but in future campaigns on-site measurements should be included to compute $\vec{L}_{h}^{G}$.

The above discussion shows that tomography provides a good spatio-temporal representation of the troposphere, consistent with a finer interpretation of the gradient analysis, and is able to better observe and quantify atmospheric phenomena which can be relevant in meteorological studies, such as the formation of a layer close to saturation above the atmospheric boundary layer.

\section{Comparison of GPS Tomography with Ra- diosonde Data}

A more conclusive example of the capabilities of GPSbased tomography is the comparison of the vertical profiles obtained from radiosonde launches at Landvetter airport (Goteborg, Sweden), about $37 \mathrm{~km}$ from the Onsala IGS site, with the tomographic solution for the launch location and time. The refractivity $N_{w}^{r s}$ is computed using the pressure, $P$, temperature, $T$, and dew-point $T_{d}$ data from the radiosonde and applying the expression $r_{r s}=m\left(P, T_{d}\right)=\frac{e_{c}\left(T_{d}\right) \epsilon}{P-(1-\epsilon) e_{c}\left(T_{d}\right)}$ to obtain the mixing ratio $r_{r s}$. Then $P_{w}^{r s}=\frac{P \cdot r_{r s}}{\epsilon}$ is the water vapor pressure ( $\epsilon=0.622)$ (see e.g. Houghton, 1977), which finally provides us with the wet refractivity $N_{w}^{r s}(T, P)=$ $3.776 \cdot 10^{5} P_{w}^{r s} / T^{2}$. In order to be consistent with the tomographic layers, radiosonde data have been averaged every $400 \mathrm{~m}$. In the horizontal direction, however, there has been no smoothing of the radiosonde data; in addition, the tomographic solution has a temporal smoothing constraint given by the Kalman filtering. Results are presented in Fig. 5 for 12:00 UTC and 18:00 UTC showing that there is a good agreement of the temporal evolution: at 12:00 UTC both methodologies show a relative maximum of wet refractivity around $800 \mathrm{~m}$, though the radiosonde presents a larger value and a wider hump; at 18:00 UTC the saturated layer has disappeared and the profile in both cases is rather uniform. The lower values produced by the tomographic solution are due to the fact that a radiosonde captures instantaneous and punctual profiles of the atmosphere, while the tomographic process combines data from a broader space through the spatial smoothing and from a longer time interval through the Kalman filtering; in addition the radiosonde senses, in this case, an area far from the core of the network, which weakens the solution there.

However, the qualitative and quantitative agreement between both solutions, as well as the description inferred from
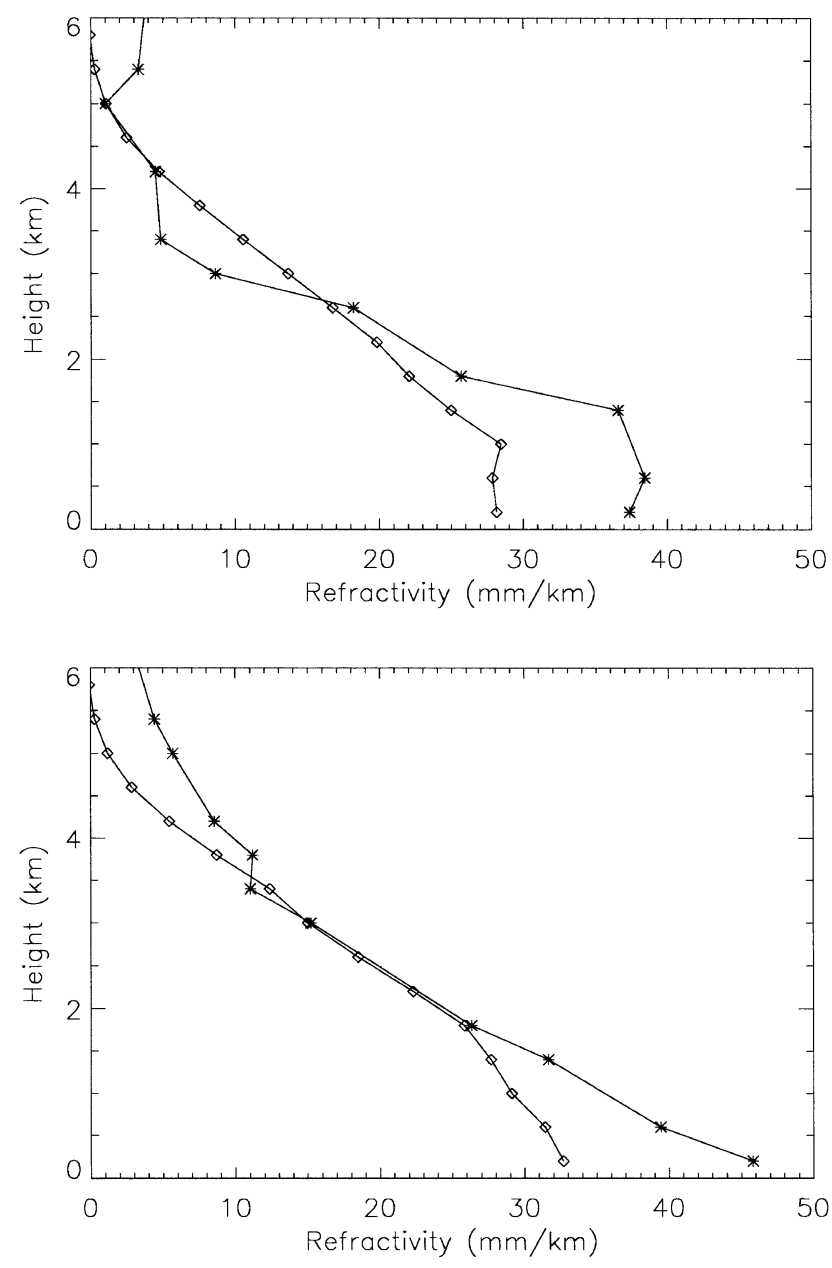

Fig. 5. Comparison of profiles observed by the radiosonde at Goteborg Airport (solid lines) and the vertical profile from the tomography analysis (diamonds) in wet refractivity at 12:00 UTC (top) and 18:00 UTC (bottom).

the analysis being consistent with the evolution of the tomographic solution, show that tomography is providing real spatio-temporal structures. The level of agreement of this experiment cannot be conclusive, given the reduced number of stations and the availability of pressure data from one location only. In fact, having appropriate ground data, these could be used to retrieval of thetomographic solution.

\section{Conclusions}

We have conducted a tomographic analysis of a smallscale GPS campaign. Using the locations of the seven GPS receivers deployed in the summer of 1998 and the GPS satellite orbits for August 24, we have performed tomographic simulations using a $3 \mathrm{D}$ refractivity field. The results provided evidence that tomography is a potential tool to describe the spatio-temporal structure of the refractivity. We used the data of August 24 because by using novel techniques we had spotted a complex meteorological effect occuring over the network. Experimental data were then used in the tomographic approach and the results were compared with radiosonde data obtained from the Landvetter airport. The agreement between both solutions was very good. Therefore, we can conclude that tropospheric tomography is feasible 
even when using a reduced set of stations. Future developements should consider the deployment of more stations and ancillary measurements to compare with.

\section{References}

Bar-Sever, Y. E., P. M. Kroger, and J. A. Borjesson, Estimating horizontal gradients of tropospheric path delay with a single GPS receiver, $J$. Geophys. Res., 103, 5019-5035, 1998.

Bevis, M., S. Businger, T. A. Herring, C. Rocken, R. A. Anthes, and R. Ware, GPS meteorology: Remote sensing of atmospheric water vapor using the global positioning system, J. Geophys. Res., 97(D14), 1578715801, 1992.

Davis, J. L., G. Elgered, A. E. Niell, and C. E. Kuehn, Ground-based measurement of gradients in the wet radio refractivity of air, Radio Sci., 28(6), 1003-1038, 1993.

Elgered, G., J. L. Davis, T. A. Herring, and I. I. Shapiro, Geodesy by radio interferometry: Water vapor radiometry for estimation of the wet delay, J. Geophys. Res., 96(B4), 6541-6555, 1991.

Elósegui, P., J. L. Davis, L. P. Gradinarsky, G. Elgered, J. M. Johansson, D. A. Tahmoush, and A. Rius, Sensing atmospheric structure using smallscale space geodetic networks, Geophys. Res. Lett., 26, 2445-2448, 1999.

Flores, A., Local tropospheric tomography software (LOTTOS) documentation, Technical report, IEEC, 1999.

Flores, A., G. Ruffini, and A. Rius, 4D tropospheric tomography using GPS slant wet delays, Annales Geophysicae, 18, 223-234, 2000.
Herring, T. A., J. L. Davis, and I. I. Shapiro, Geodesy by radio interferometry: The application of kalman filtering to the analysis of very long baseline interferometry data, J. Geophys. Res., 95(B8), 12561-12581, 1990.

Houghton, J.T., The Physics of Atmospheres, 203 pp., Cambridge University Press, 1977.

Kursinski, E. R., The GPS Radio Occultation Concept: Theoretical Performance and Initial Results, Ph.D. thesis, California Institute of Technology, 288 pp., 1997.

Press, W. H., S. A. Teukolsky, W. T. Vetterling, and B. P. Flannery, Numerical Recipes in Fortran. The Art of Scientific Computing, 961 pp., Cambridge university Press, second edition, 1992.

Ruffini, G., A. Flores, and A. Rius, GPS tomography of the ionospheric electron content with a correlation functional, IEEE Transactions on Geoscience and Remote Sensing, 36(1), 1998.

Ruffini, G., L. P. Kruse, A. Rius, B. Bürki, L. Cucurull, and A. Flores, Estimation of tropospheric zenith delay and gradients over the Madrid area using GPS and WVR data, Geophys. Res. Lett., 26(4), 447-450, 1999.

Thayer, G. D., An improved equation for the radio refractive index of air, Radio Sci., 9(10), 803-807, 1974.

Webb, F. H. and J. F. Zumberge, An Introduction to GIPSY-OASIS II, Jet Propulsion Laboratory, California Institute of Technology, 1997.

A. Flores (e-mail: flores@ieee.org), L. P. Gradinarsky, P. Elósegui, G. Elgered, J. L. Davis, and A. Rius 